\title{
REVISTA HISTORIA DE LA EDUCACION LATINOAMERICANA No 16
}

\author{
Diana Soto Arango, Nina Lluhi de Hasegawa
}

Tunja, Tokio, 30 de junio del 2011

Esta publicación se ha dedicado al tema monográfico de los textos escolares. La relevancia del tema la asumimos por las investigaciones que sobre este contenido viene desarrollando un equipo desde Francia y España. Esta red presenta vínculos con investigadores, en América Latina, donde podemos destacar en Venezuela al Dr. José Pascual Mora, presidente de la Sociedad de Historia de la Educación Latinoamericana. Pero hay que decir que principalmente, se motivó el tema monográfico, por la investigación que realizó el Grupo de investigación: "Historia

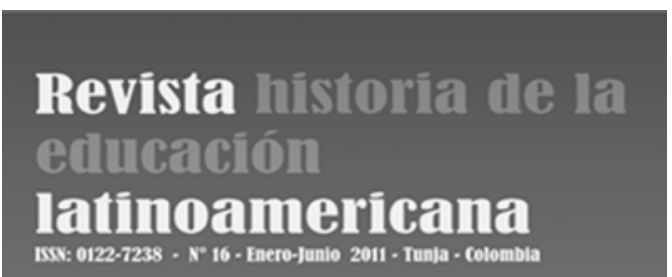
y Prospectiva de la Universidad Latinoamericana"- HISULA, sobre "La independencia americana: textos enseñanza e imaginarios escolares en Colombia y España. 2009 a 2011".

En este estudio se analizó a través de los textos escolares y las formas como el tema de la independencia americana, desde el aula escolar, con los contenidos, mensajes, iconografía de los textos, guías y procesos de enseñanza aprendizaje, han originado unos imaginarios sociales en los estudiantes de educación básica primaria y secundaria que asimilan hacia una nueva identidad en el imaginario de la nación en la región cundí-boyacense de Colombia, y en 2 ESO y 4 Bachillerato de la Provincia de Andalucía, España.

Por otra parte, era relevante destacar a un educador que hubiese incidido con sus textos en la educación de América Latina y el Caribe. El Comité Editorial asumió para este número de la Revista al educador ecuatoriano Miguel Febres Cordero. Este educador, ingresa a los Hermanos cristianos 
con el nombre de G. M. Bruño. Desde allí, se da a conocer como uno de los autores de textos escolares de mayor influencia, en este continente americano de habla hispana, desde finales del siglo XIX y en la primera mitad del siglo XX. Quizá, como lo indica en el artículo, el Dr. Javier Ocampo, "Se recuerdan los textos de Aritmética, Álgebra y Geometría del Hermano Cristiano G.M. Bruño, que formaron a los colombianos, ecuatorianos y de otros países de Hispanoamérica". Es así que, con más de cincuenta textos referentes a lenguaje, español y literatura, ortografía, física, botánica, religión, historia sagrada, tabla de logaritmos, contabilidad, y otros incidió en el aprendizaje de miles de niños y jóvenes de América Latina y el Caribe que asumieron como propios, en las aulas de clase, la colección de textos de G.M. Bruño.

Los artículos presentados en este número, sobre textos escolares, se orientan en primera instancia a las diferentes formas de influencia, de la religión católica, en los sistemas de enseñanza a través de los textos escolares, desde el siglo XIX y XX. Como es lógico, el primer escrito, es el referente a Miguel Febres Cordero que de la mano del Dr. Javier Ocampo iniciamos este recorrido en la revista con el educador identificado como G.M. Bruño y la influencia de los hermanos cristianos en el contexto de América Latina y el Caribe..

Indudablemente, este trabajo que presentamos a la comunidad académica Iberoamericana no hubiese sido posible sin la especial colaboración de la Dra. Nina Lluhi de Hasegawa, directora del Instituto Iberoamericano de Sophia University en el Japón quien nos acompaño en la función de la Coordinación editorial de este número. A los evaluadores quienes de manera filantrópica nos apoyan en este proceso editorial. Igualmente, agradecemos la labor de la joven investigadora Sandra Liliana Bernal Villate de Grupo de investigación: "Historia y Prospectiva de la Universidad Latinoamericana"- HISULA y de la profesora Doris Torres por el apoyo continuo en esta tarea de visibilizar los productos de las investigaciones. Al Doctorado Ciencias de la Educación de la Universidad Pedagógica y Tecnológica de Colombia por la financiación de la misma.

Finalmente, debo señalar que dadas las condiciones de exigencia en los indicadores internacionales, de las revistas indexadas como es el caso de la Revista Historia de la Educación Latinoamericana, a partir del número 15 se retoma la versión de todos los artículos en inglés que se editarán en la modalidad electrónica establecida en los repositorios de la web de la revista, de RUDECOLOMBIA y de la Sociedad de Historia de la Educación Latinoamericana. Por lo tanto, los autores una vez se les apruebe la versión presentada en español deben entregar la versión inglesa para poder ser editado el artículo.

Palobra No. 13. Agosto de 2013 